\title{
Setting up an outpatient service for early medical termination
}

\author{
Cathy Tupper, Shonagh Speed Andrews
}

\begin{abstract}
Objectives In parts of the UK, demand for termination of pregnancy exceeds local availability on the National Health Service. The study objective was to report on setting up and running a new outpatient service for early medical termination under 7 weeks' gestation.
\end{abstract}

Methods We describe the process of setting up the clinic within the confines of the Abortion Act 1967, the training of staff, acquisition of resources, cost analysis and negotiations with the Primary Care Trust. We report on running the service in the first 12 months, difficulties encountered and how these have been overcome. Our experience is compared with the limited published data available.

Results Of 171 patients referred in the first year, 148 were offered an appointment and 100 women completed outpatient treatment for medical termination under 7

\section{Introduction}

In many areas in the UK, requests for termination of pregnancy (TOP) exceed the willingness and capability of local hospitals to provide them. As a consultant in family planning with 10 years' experience in this locality, one of the authors (CT) has witnessed a rise in waiting times for surgical termination to up to 4 weeks on occasion. At the same time, demands on general gynaecology have resulted in fewer TOPs being provided; often only one method of termination is offered and strict criteria applied, for example, an upper gestation limit of 12 weeks. This has resulted in patients having to travel considerable distances from our outlying area to other provider units, albeit National Health Service (NHS) funded.

In the Morecambe Bay Primary Care Trust (PCT) area in $2004,33 \%$ of women were unable to obtain a termination within local NHS hospitals because of inadequate capacity. ${ }^{1}$ Because of the relative distance of this area from urban centres, this resulted in women being required to travel over 200 miles (return trip) to the nearest outside agency for treatment. Both the local hospitals trust and the contracted outside agency provided surgical termination only.

This service was developed in order to:

- Address the Royal College of Obstetricians and Gynaecologists (RCOG) guidance on providing patient choice of any method of termination at any gestation. ${ }^{2}$ Only surgical termination was previously offered in this area.

- Provide a safe outpatient medical termination for gestations under 49 days.

\section{Contraception Clinic, Atkinson Health Centre, Barrow-in-Furness, UK \\ Cathy Tupper, FFFP, Consultant in Family Planning and Reproductive Health Care \\ Shonagh Speed Andrews, MRCGP, DFFP, General Practitioner and Staff Grade in Family Planning}

Correspondence to: Dr Cathy Tupper, Atkinson Health Centre Market Street, Barrow-in-Furness LA14 2LR, UK.

E-mail: cathy.tupper@cumbriapct.nhs.uk weeks' gestation. $90 \%$ of patients attended follow-up at 2 weeks and the remainder were contacted by telephone. Clinical outcomes were similar to those reported in the literature apart from initially higher numbers of women undergoing evacuation of retained products of conception. The estimated cost per case in the first year was $£ 156$, which represented a considerable cost saving compared to $£ 498$ for surgical termination and $£ 423$ for inpatient medical termination.

Conclusions Our service is successful, viable for the future and has acceptably good patient outcomes, which are improving all the time.

Keywords outpatient, service delivery, termination of pregnancy

J Fam Plann Reprod Health Care 2007; 33(3): 199-202

(Accepted 26 April 2007)

\section{Key message points}

- Small services can provide a significant contribution to termination of pregnancy services, providing choice and follow-up contraception care.

- With backup and support, medical termination patients can be treated as outpatients.

- This service is cost effective and the training and equipment needed benefit other patients.

- Provide an accessible service locally, which was only otherwise available out of area via a private provider.

- To supplement the surgical termination service, which is currently struggling to provide surgical terminations under the local gestational limit of 12 weeks.

- To meet the Department of Health targets on TOP (reduced waiting times and increased number provided under 10 weeks' gestation).

- To free up gynaecology beds, which are needed for other patients, in order to reduce patient waiting times.

- Contribute towards cost savings in the NHS budget.

It is well recognised that medical TOP undertaken at less than 63 days' gestation involving administration of mifepristone and misoprostol 48 hours apart ${ }^{3-11}$ is effective, safe and cost-effective. However, bed occupancy with nurse supervision is a constraining factor in the number of women able to access this option. (Gestations greater than 7 weeks may also require a second dose of misoprostol.) Women under 7 weeks' gestation are less likely to bleed heavily or require analgesia, ${ }^{3-6}$ either of which could necessitate admission. Also the complete abortion rate is higher under 49 days' gestation, again avoiding possible surgical intervention. ${ }^{7-11}$

Some of the large abortion charities had already started outpatient treatment as an option for women in very early pregnancy; in one reported study from the UK women were able to self-administer misoprostol at home. ${ }^{12}$ Whilst highly acceptable to women, this may lie outside the provisions of the Abortion Act 1967 since treatment was not being administered in licensed premises. As all misoprostol given as an abortifacient is outside the product licence but is widely accepted practice, action must be 
Table 1 Set-up and annual additional running costs of the early medical termination service

\begin{tabular}{lr}
\hline Item & Cost $(£)$ \\
\hline $\begin{array}{l}\text { Set-up costs (initial) } \\
\text { Portable scanner with abdominal and transvaginal } \\
\text { probes }\end{array}$ & 23500 \\
Training (including travel) for two team members & 3000 \\
& \\
Running costs (per year) & 4250 \\
Staff grade: one session per week & 4000 \\
Nursing & 2928 \\
Administration: one extra session & 1450 \\
Drug costs & 3000 \\
Service contract for scanner & 15628 \\
& 156 \\
\hline Total running costs & \\
Estimated cost per case (three per week) &
\end{tabular}

taken to redress this discrepancy to the benefit and desire of the pregnant women. In consultation with local gynaecology colleagues, it was felt that this service could be offered from the contraceptive service, with backup from the gynaecology department. The service was in demand from the start and has now had to be expanded. It is now able to offer treatment for virtually all women referred at appropriate gestation.

The service commenced on April 2005. This article documents the first 100 women proceeding with an outpatient medical termination.

\section{Methods}

The scheme had the unreserved support of gynaecology colleagues. The location was in an existing community contraceptive clinic on a hospital site already registered under the terms of the Abortion Act 1967, which was located midway between the two district general hospitals providing surgical TOP and midway between the largest population centres of the PCT.

In this service women are treated at gestations of less than 7 weeks only. This makes it possible to give a single dose of misoprostol (48 hours after mifepristone) as an outpatient, the patient then returning home to abort the pregnancy. All women are reviewed 2 weeks later, with ultrasound used to confirm that the treatment has been effective.

\section{Business plan}

Based on an existing clinic, staff hours were increased, and another doctor employed to provide continuity of service. The expense of increased pathology and haematology tests are covered in the PCT block contract. The cost of misoprostol, mifepristone and anti-D covering the national incidence of $15 \%$ was included. These costs were formulated into a business plan with the sexual health commissioner for the PCT and presented to the PCT board.

Table 2 Annual cost of the early medical termination service over its first 5 years of operation

\begin{tabular}{lr}
\hline Item & Cost (£) \\
\hline Ultrasound scanner & 5000 \\
Maintenance/insurance contract & 3000 \\
Staff & 11000 \\
Drug costs & 1450 \\
Staff training & 600 \\
Total cost & 21050 \\
Cost per case (average five patients & 84 \\
per week for 50 weeks) & \\
\hline
\end{tabular}

This provided a significant cost saving to the PCT compared to the purchase of service from other providers. The largest set-up cost was that of the ultrasound scanner. This was obtained via a bid to the Department of Health under the auspices of improvement of TOP services.

Based on the figures in Table 1, the calculated additional cost per case was $£ 156$. This compares most favourably to the $£ 498$ per case on 'Payment by Results' for surgical termination and $£ 423$ for medical termination. Costs for blood tests, other treatments and routine clinic costs are already covered in the block contract for family planning services.

Table 2 projects the cost per annum for the first 5 years of running the service and the significantly reduced cost per case based on seeing a minimum of five cases per week, which is now the case in our clinic.

\section{Staff training requirements}

All staff members were initially asked if they objected to working with or treating women requesting termination. No objections were recorded. Some staff members were already working with women requesting termination. Others attended a day course on specific counselling skills. Two staff attended a specifically designed course for practical skills in transvaginal ultrasound scanning; neither had any previous practical scanning skills. The third clinical member will be trained in house as and when funds allow. The RCOG standards and competences in providing abortion services are being observed. ${ }^{1}$

\section{Setting up the service/clinic process}

The various stages involved in setting up the service and an outline of the clinic process are detailed in Boxes 1 and 2.

\section{Results}

Table 3 shows all the referrals received in the first year of operation of the service from April 2005 to March 2006. Most of the patients who could not be offered an appointment were referred in the first 8 months. All available slots were filled from the first week of the service opening; as a result the service was extended to provide six new weekly appointments. Many fewer referrals are now turned away but this is continuously monitored.

\section{Box 1: Setting up the service}

- All general practitioners (GPs) were informed of the new service. GPs and patients were made aware that this was an outpatient service. Patients without support at home after the second treatment were deemed unsuitable candidates for the service.

- Administration staff at an existing family planning clinic received all referrals on a single booking telephone number (no faxes). (One additional half day of administration was required to receive Monday-Friday morning referrals.)

- GPs were provided with an action flow chart and patient care pathway and an information sheet for patients on medical termination.

- On receiving the referral, the administrator first confirms that the patient would be less than 7 weeks' gestation by the time of their initial appointment using the last menstrual period and gestation calculator. [NB. This is essential in order to avoid inappropriate referrals that may introduce delay for another patient in what is a rapid response service.]

- The GP is given the date and time of the outpatient appointment when the telephone referral is received. The patient brings the referral letter, signed HSA1 (blue form) and a note of their blood group (if known) to the first appointment.

- Referrals from the administrator are e-mailed on the morning of the clinic. 


\section{Box 2: Outline of the clinic process}

\section{First appointment}

- Sexual and contraceptive history taken.

- Future contraception choices and method agreed and prescribed if possible.

- Cervical swab for chlamydia, high vaginal swab and other clinically indicated tests (positive sexually transmitted infections are treated and referred to genitourinary medicine for contact tracing)

- Blood group checked if unknown. (GPs are asked to provide this information, if available, to decrease the necessity for taking blood samples.)

- Transvaginal ultrasound scan done to confirm gestation and intrauterine pregnancy.

- HSA1 signed by GP, countersigned in clinic.

- After counselling, a standard NHS consent form completed for medical TOP.

- Patient witnessed to take $200 \mathrm{mg}$ mifepristone orally.

- Patient provided with a second information sheet that details for the patient and any other health professionals what action to take if problems arise.

- Advice is given to the patient regarding providing themselves with sanitary towels and simple analgesia or ibuprofen for the second treatment.

- The need for a companion for the second treatment is again stressed.

- Copy of consent given to patient and follow-up appointments made.

- Misoprostol (and anti-D if required) prescribed to enable administration by the nurse 48 hours later.

- Gynaecology ward informed of the names of patients undergoing treatment.

- Telephone backup is available 24 hours a day, provided in the daytime by the clinic staff and out of hours by the gynaecology ward staff at the nearest district general hospital. (NB. Very few calls have been received.)

\section{Second appointment}

- Two days later the patient attends for about 10 minutes, again as an outpatient, for the second treatment at one of three hospitals, whichever is closest to the patient's home.

- $800 \mu \mathrm{g}$ misoprostol administered vaginally. Anti-D is given if necessary. The backup information is reiterated.

- The patient keeps a copy of her treatment sheet in case medical advice needs to be sought.

- Oral or injectable contraception is initiated.

\section{Third appointment}

- The follow-up appointment is 14 days after the first treatment.

- History taken.

- Swab results given, treatment given and referral to genitourinary medicine as necessary.

- Transvaginal ultrasound scan done to exclude ongoing pregnancy and quantify any retained products.

- Subdermal and intrauterine contraception are inserted if requested at this appointment, the latter only if the uterus is empty on transvaginal ultrasound scanning.

Table 4 shows the clinical outcomes and future contraceptive choices of the 100 patients who completed treatment in the first 12 months that the service operated.

One patient underwent evacuation of the retained products of conception (ERPC) on the day of the misoprostol treatment on clinical grounds because of profuse vaginal bleeding. She did not require blood transfusion. Another seven patients were offered ERPC

Table 3 Referrals to the early medical termination service in the first 12 months of operation

\begin{tabular}{lr}
\hline Outcome & Patients $(\boldsymbol{n})$ \\
\hline Total number of referrals & 171 \\
Patients who could not be offered an appointment & 23 \\
Did not attend or cancelled last minute & 17 \\
Attended but were found to have miscarried & 12 \\
Patients over 7 weeks' gestation & 17 \\
Did not go through treatment after counselling & 2 \\
Completed the treatment & 100 \\
\hline
\end{tabular}

Table 4 Clinical outcomes in the first year

\begin{tabular}{lr}
\hline Parameter & Patients $(\boldsymbol{n})$ \\
\hline Outcome & \\
Patients completed treatment in the first 12 months & 100 \\
Did not attend follow-up & 10 \\
Had no complications & 67 \\
Had or required surgical evacuation of retained & 8 \\
products of conception & \\
Ongoing pregnancy & 1 \\
Women found to have products of conception & 14 \\
within the uterus at follow-up $<3 \times 3 \mathrm{~cm}$ & \\
Chlamydia positive & 4 \\
Bacterial vaginosis & 10 \\
Type of future contraception & \\
Intrauterine system & 10 \\
Intrauterine device & 7 \\
Implant & 5 \\
Injectable & 11 \\
Oral contraceptive pill & 51 \\
Vasectomy & 1 \\
No method (no partner) & 5 \\
Unknown (failed follow-up) & 10 \\
\hline
\end{tabular}

after retained products were seen at follow-up. In line with our local gynaecological department policy, the 14 women with retained products less than $3 \times 3 \mathrm{~cm}$ on transvaginal ultrasound were treated expectantly. These figures are higher than other data published for women under 49 days' gestation, and as they were mostly in the first few months of the service being set up they probably reflect our own learning curve (i.e. increased scanning competence and the benefit of hindsight).

The patients who failed to attend for follow-up were mainly from the further reaches of our large geographical area. All were contacted by telephone and advised to seek assessment by their GP to ensure that treatment had been successful.

Any identified sexually transmitted infections were treated at the 2-week follow-up appointment; women with a positive chlamydia test were referred to genitourinary medicine for contact tracing.

Patients treated by us were encouraged to use longacting reversible contraception (LARC) as women who have had a TOP are known to be at greater risk of a further unplanned pregnancy. ${ }^{2}$ The future contraceptive plans of the women are outlined in Table 4; $33 \%$ of them chose LARC. Women were actively dissuaded from using condoms alone as contraception.

A number of problems were encountered that required action and changes in procedure as detailed below.

- Initially with only one member of staff trained to use ultrasound and only one doctor, the service could not offer continuity.

- A patient who fails to attend for follow-up is contacted by telephone and letter to urge her to attend. The woman's GP is informed by telephone.

- Repeat pregnancy tests at follow-up are rarely negative and are therefore not a reliable indicator.

- Patients who fail to attend the initial appointment are contacted by telephone at the number given by the GP on referral, and the GP informed of non-attendance

- Despite repeated advice on the importance of having a companion on the day of the second treatment, some patients attend alone and in one case a patient was found waiting for a bus.

- If ultrasound cannot confirm an intrauterine pregnancy and the dates suggest gestation $<5$ weeks the patient is invited back the following week. If ectopic pregnancy is suspected then direct referral is made to gynaecology. 


\section{Discussion}

Previous articles discuss the early results of newly set-up clinics for medical termination ${ }^{13,14}$ and whilst their results are given, together with some drug cost analysis, there is no blueprint to be copied nor staff costing published. Other investigators $12,15,16$ have demedicalised medical termination by allowing the patient to self-treat with misoprostol at home rather than in hospital. Under the terms of the Abortion Act 1967, all treatment for abortion should be given in licensed premises. We believe that our service, whilst still remaining within the law, provides the least medical input possible.

Our results for patients requiring ERPC are higher than those published for patients less than 49 days' gestation. $6,12,17,18$ This may reflect the small numbers of patients seen so far and our own learning curve. The uptake of telephone advice has amounted to a handful of calls and only one patient was admitted on the day of the misoprostol treatment. Our follow-up non-attendance rate is disappointing compared to others; ${ }^{12,13}$ however, to our knowledge none of these patients had complications or needed admission and the majority of these patients were from the furthest reaches of the wide geographical area covered by Morecambe Bay PCT . Better uptake of LARC has been achieved, 17 and our data resemble those from other studies. 13

Many aspects of the work (e.g. receiving referrals) were taken on within existing staffing time. The nursing telephone support to patients undergoing medical termination has allowed other patients to access advice outside our clinic times. The availability of an ultrasound scanner for use in the other clinics has also decreased referrals to the early pregnancy assessment unit and the ultrasound department. For patients it may avoid a 4-6-week wait for routine ultrasound for location of intrauterine contraceptive devices with missing threads.

\section{Conclusions}

We conclude that with the appropriate support and training, community contraception clinics can provide an appropriate setting for an early medical termination of pregnancy service with minimal medical intervention. In the authors' own geographical area it has provided a choice of methods not previously available and has prevented the necessity of long journeys to access some providers. This can be a cost-effective service for the local PCT even in a small service such as the one described here, and it can have a positive spin-off for many other patients.

Having a site in an already appropriately registered hospital made the process possible, as did the availability of a Department of Health grant for purchasing the ultrasound scanner.

In the near future we aim to trial a patient satisfaction survey and explore the possibility of same-day treatment with mifepristone and misoprostol. ${ }^{19}$
Acknowledgements

The authors wish to thank the personnel working in the contraception service for their positive attitude to this new service and the patients who have used it to date.

Statements on funding and competing interests

Funding None identified.

Competing interests Cathy Tupper is an ad hoc trainer and lecturer for Organon.

\section{References}

1 Government Statistical Service. Abortion Statistics, England and Wales: 2004 (Statistical Bulletin 2005/11). London, UK: Department of Health, 2005

2 Royal College of Obstetricians and Gynaecologists (RCOG). The Care of Women Requesting Induced Abortion (National Evidence-Based Clinical Guideline No. 7). London, UK: RCOG Press, 2004.

3 Schaff EA, Fielding SL, Eisinger SH, Stadalius LS, Fuller L. Low dose mifepristone followed by vaginal misoprostol at 48 hours for abortion up to 63 days. Contraception 2000; 61: 41-46.

4 Rodger MW, Baird DT. Blood loss following induction of early abortion using mifepristone (RU486) and a prostaglandin analogue (gemeprost). Contraception 1989; 40: 439-447.

5 Prasad RN, Choolani M, Roy A, Ratnam SS. Blood loss in termination of early pregnancy with mifepristone and gemeprost. Aust N Z J Obstet Gynaecol 1995; 35: 329-331.

6 Spitz IM, Bardin CW, Benton L, Robbins A. Early pregnancy termination with mifepristone and misoprostol. $N$ Engl J Med 1998; 338: 1241-1247.

7 McKinley C, Thong KJ, Baird DT. The effect of dose of mifepristone and gestation on the efficacy of medical abortion with mifepristone and misoprostol. Hum Reprod 1993; 8: 1502-1505

8 Ashok PW, Penney GC, Flett GM, Templeton A. An effective regimen for medical abortion: a report of 2000 consecutive cases. Hum Reprod 1998; 13: 2962-2965.

9 Creinin MD, Vittinghoff E, Keder L, Darney PD, Tiller G. Methotrexate and misoprostol for early abortion: a multicentre trial. 1. Safety and efficacy. Contraception 1996; 57: 321-327.

10 Kahn JG, Becker BJ, Maclsaa L, Amory JK, Neuhaus J, Olkin I, et al. The efficacy of medical abortion: a meta-analysis. Contraception 2000; 61: 29-40.

11 Randel J, French L. Mifepristone and misoprostol for termination of early pregnancy. J Fam Pract 1998; 47: 96-97.

12 Hamoda H, Ashok P, Flett GMM, Templeton A.. Home selfadministration of misoprostol for medical abortion up to 56 days gestation. J Fam Plann Reprod Health Care 2005; 31: 189-192.

13 Ojidu JI, Sabharwal SD. Setting up a one-stop mifepristonemisoprostol medical termination of pregnancy service for all gestations from 5 to 23 weeks - a review of 482 cases. J Obstet Gynaecol 2001; 21: 386-388.

14 Suhonen S, Heikinheimo O, Tikka M, Haukkamaa M. The learning curve is rapid in medical termination of pregnancy first year results from the Helsinki area. Contraception 2003; 67: 223-227.

15 Kiran U, Amin P, Penketh RJ. Self-administration of misoprostol for termination of pregnancy: safety and efficacy. $J$ Obste Gynaecol 2004: 24: 155-156.

16 Shannon CS, Winikoff B, Hausknecht R, Blumenthal PD, Oyer D Sankey $\mathrm{H}$, et al. Multicentre trial of a simplified mifepristone medical abortion regimen. Obstet Gynecol 2005; 105: 345-351.

17 Yassin AS, Cordwell D. Does dedicated pre-abortion contraception counselling help to improve post-abortion contraception uptake? J Fam Plann Reprod Health Care 2005; 31: 115-116.

18 Penney G, McKessock L, Rispin R, El-Refaey H, Templeton A. An effective, low cost regime for early medical abortion. $\mathrm{Br} J$ Fam Plann 1995; 21: 5-6.

19 Kapp N, Borgatta.L, Carr Ellis S, Stubblefield P. Simultaneous very low dose mifepristone and vaginal misoprostol for medical abortion. Contraception 2006; 73: 525-527. 